\title{
Optimal routing for minimum wavelength requirements in end-to-end optical burst switching rings
}

\author{
Reinaldo Vallejos ${ }^{1}$, Alejandra Zapata ${ }^{1}$, Víctor Albornoz ${ }^{2}$ \\ ${ }^{1}$ Telematics Group, Electronic Engineering Department, Universidad Técnica Federico \\ Santa María, Av. España 1680, Valparaíso, Chile \\ ${ }^{2}$ Industrial Engineering Department, Universidad Técnica Federico Santa María, Av. Santa \\ María 6400, Santiago, Chile \\ \{reinaldo.vallejos, alejandra.zapata, victor.albornoz\}@usm.cl
}

\begin{abstract}
A novel routing and link dimensioning optimisation method which minimises the total wavelength requirements of dynamic optical WDM rings the most popular topology in metropolitan networks- is proposed. The method finds the solution (set of routes) of minimum cost by solving an integer linear optimisation problem. Contrary to the common belief, results show that the optimal routes found by the proposed method are not necessarily balanced and that significant wavelength savings are achieved compared to the usual balanced-load routing approach in rings. This makes the proposed method the best choice for implementation in future dynamic WDM ring networks.
\end{abstract}

Keywords: optical burst switching networks, rings, routing, dimensioning, wavelength requirements.

\section{Introduction}

One promising dynamic WDM network architecture, given the current unavailability of optical buffers and processors (which hampers the implementation of the already successful packet-switching technique very much used in Internet), is Optical Burst Switching (OBS) [1-4]. The operation principle of OBS combines the dynamic reservation/release of optical network resources with low speed requirements for the electronic layer (in charge of buffering and processing). To do so, OBS networks electronically aggregate packets at the edge of the network into a burst. Once a burst is ready to be transmitted, corresponding optical network resources are requested. The allocation of optical network resources can be implemented in a hopby-hop [1,2] or in an end-to-end [3,4] basis. This paper focuses in the latter, as it has been shown to significantly reduce the burst loss rate with respect to a hop-by-hop OBS network of equivalent complexity [5]. Thus, only after all the resources from source to destination have been reserved the data burst is released into the optical core.

In an end-to-end OBS network each node consists of an optical switch locally connected to an edge router (see Figure 1). Every edge router, where burst aggregation is carried out, is equipped with one buffer per destination. Incoming 
packets are classified according to destination and stored in the corresponding buffer. During the burst aggregation process the resource allocation request state is triggered once a pre-defined condition is met (e.g., latency or burst size), and a request is sent through the network to reserve resources for the burst. Resource reservation can be done in a centralised or distributed way. In each case, requests are processed according to the implemented resource allocation algorithm. If such algorithm finds an available route, the network is configured to establish the end-to-end route and an acknowledgement is sent to the corresponding source node. Otherwise, the request is dropped with a NACK message sent to the source node which blocks the burst from entering the network. In this paper an end-to-end OBS network with full wavelength conversion capability is considered, as such feature is key for a dynamic network to achieve significant wavelength savings with respect to the static approach [6].

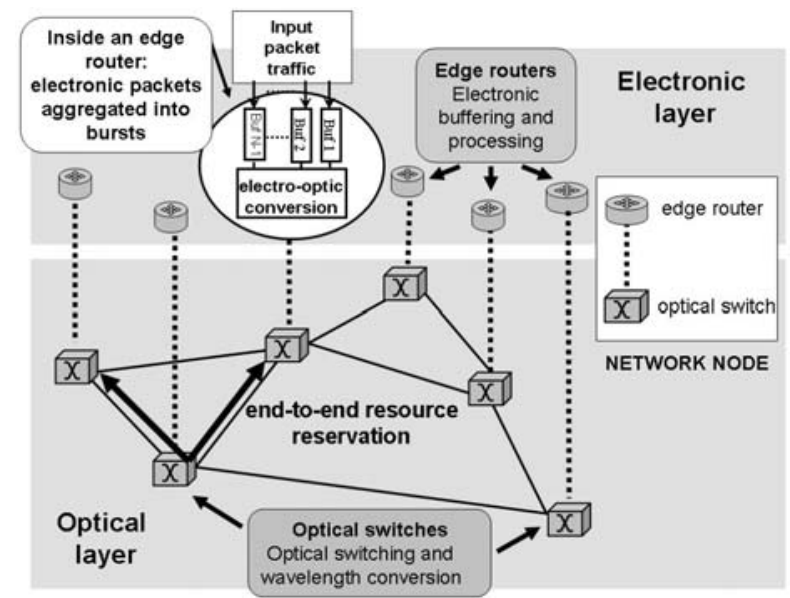

Fig. 1. Schematic of an end-to-end OBS network

Given that wavelengths are costly resources, the efficient dimensioning of an endto-end OBS network to achieve a target blocking probability is of fundamental importance. The dimensioning of a network is strongly dependant on the routing algorithm, whose aim is to determine the routes to be used by each node pair to transmit information such that minimum resources are required. A common nearoptimal routing heuristic in static WDM networks (where a route for each node pair is permanently established) has been to utilise the shortest paths whilst minimising the number of wavelengths in the most loaded link, see for example [7]. This approach (used in rings, for example, in $[5,8]$ ) leads to routes that balance the traffic load throughout the network. Later, in [9], it was shown that using these routes in the dynamic case results in significant lower blocking probability than arbitrarily selecting any shortest path for each node pair. Consequently, this balanced-load routing approach has been commonly used in dynamic networks, see for example [5, 10,11]. However, by maintaining the links uniformly loaded, the statistical multiplexing of connections per link is not fully exploited.

In this paper we propose a novel method for optimal routing and dimensioning of optical network rings, the most popular topology in metropolitan networks [12]. The 
proposed method determines - by solving an integer linear optimisation problem in an off-line manner- the set of routes (one per node pair) which minimises the total wavelength requirements whilst satisfying a given blocking probability. The resulting routes are such that (contrary to the common belief) the traffic load is not necessarily balanced among the network links and routes might be longer than the shortest paths (as long as the wavelength savings due to the statistical multiplexing, because of the traffic load concentration, are higher than the additional wavelengths required by the extra hops of possibly longer routes).

For dynamic ring operation, the obtained routes are stored in a routing table and looked for when connections must be established on demand (that is, a fixed routing dynamic algorithm is used).

The remaining of this paper is as follows: section 2 describes the optimisation method; section 3 presents the numerical results whilst section 4 summarises the paper.

\section{Optimal routing and link dimensioning method}

\subsection{Network and traffic model}

The network is represented by a directed graph $G=(\mathcal{N}, \mathcal{L})$ where $\mathcal{N}$ is the set of network nodes and $\mathcal{L}$ the set of unidirectional links (adjacent nodes are assumed connected by two fibres, one per direction). The cardinality of the sets $\mathcal{N}$ and $\mathcal{L}$ are denoted by $N$ and $L$, respectively.

The burst aggregation process transforms the original packet traffic into a burst traffic which can be described by a source which switches its level of activity between two states: ON (burst transmission) and OFF (time between the end of the transmission of a burst and the beginning of the following one). During the ON period, the source transmits at the maximum bit rate (corresponding to the transmission rate of one wavelength). During the OFF period, the source refrains from transmitting data. This behaviour has been modelled by an ON-OFF alternating renewal process, for example in [13-15], and it is also used in this paper to characterise the burst traffic generated at the edge nodes.

In the network there are $N(N-1)$ different connections (source-destination pairs). In this paper, each connection is mapped to a number -denoted by $c$ - between 1 and $N(N-1)$. Each connection $c$ is associated to a route, $r_{c}$, determined by a fixed routing algorithm and established only during the ON period of connection $c$.

According to the usual characterization of an ON-OFF traffic source [16], the demand of connection $c$ is defined by two parameters: mean OFF period duration (denoted by $t_{O F F}$ ) and mean $\mathrm{ON}$ period duration (denoted by $t_{O N}$ ), equal for all connections (homogeneous traffic). The mean ON period includes burst transmission as well as the time required to reserve/release resources for the burst. The latter depends on the reservation mechanism used, which is out of the scope of this paper. According to the alternating renewal process theory [17], the traffic load offered by 
one connection, $\rho$, is given by $t_{O N} /\left(t_{O N}+t_{O F F}\right)$. The traffic load can be also though as the stationary state probability that a given connection is in ON state.

\subsection{Routing and link dimensioning optimal method}

In a network ring a routing algorithm must determine, for each node pair, whether the clockwise or the counter-clockwise route is used. Given a specific routing algorithm (i.e. the route for each node pair has been determined), the network cost $C$ (in terms of number of wavelengths) is given by the sum of the wavelength requirements of all the network links. That is:

$$
C=\sum_{l \in \mathcal{L}} W_{l}
$$

where $W_{l}$ denotes the number of wavelengths required in each link $l \in \mathcal{L}$ such that a target blocking probability is guaranteed (in an end-to-end OBS network bursts are blocked from entering the network due to unavailability of network resources as opposed to the conventional hop-by-hop OBS networks where bursts are lost at any point along the path due to wavelength contention).

\subsubsection{Evaluation of $\boldsymbol{W}_{l}$}

The evaluation of $W_{l}$ is divided in two steps: firstly, the target value of the blocking of each network link is determined (Eqs. (2)-(4) in the following); then, this target value (obtained in Eq. (4)) is used to obtain the value of $W_{l}$ from an analytical expression that relates $W_{l}$ with the link blocking probability (Eqs. (5)-(6) in the following).

\section{Target value for the link blocking, $B_{l}$}

The number of wavelengths that the link $l$ must be equipped with, $W_{l}$, depends on the level of acceptable blocking probability for such link, $B_{l}$ (for example, in the extreme case of $B_{l}=0, W_{l}$ must be equal to the number of connections using the link $\left.l, N_{l}\right)$. $B_{l}$ can be easily determined from the value of the blocking specified for the network connections according to the following expression (assuming link blocking independence):

$$
B_{c}=1-\prod_{\forall l \in r_{c}}\left(1-B_{l}\right)
$$

where $B_{c}$ is the blocking probability of connection $c$ and $l$ denotes a link in the route $r_{c}$ associated to connection $c$.

Let $H_{c}$ be the length (in number of hops) of the route $r_{c}$. There are many values for each factor (of the form $\left(1-B_{l}\right), \forall l \in \mathcal{L}$, in the right side of Eq. (2)) which satisfy the equality of Eq. (2), including the one where all the $H_{c}$ factors of Eq. (2) have the same value. To facilitate analytical treatment of Eq. (2) without modifying the target of the dimensioning process (to guarantee that the blocking $B_{c}, 1 \leq c \leq N(N-1)$, does not exceed a given value $B$ ) the same value of $B_{l}$ for all $H_{c}$ links in the route $r_{c}$ is used. This leads to the following expression: 


$$
1-\left(1-B_{l}\right)^{H_{c}} \leq B ; \quad 1 \leq c \leq N(N-1)
$$

where $H_{c}$ is the length (in number of hops) of the route $r_{c}$.

Because the different connections using a given link $l$ might have different route lengths, to guarantee that all connections (even the longest) achieve a blocking probability lower than $B$, the value of $H_{c}$ corresponding to the longest route using link $l$ is used in Eq. (3). Thus, the link $l$ must be dimensioned so the following condition is met:

$$
B_{l}=1-\sqrt[\hat{H}_{1}]{(1-B)} ; \quad \forall l \in \mathcal{L}
$$

where $\hat{H}_{l}$ corresponds to the length (in number of hops) of the longest route using link $l$.

\section{Evaluation of $W_{l}$}

Eq.(4) provides a target value for the link blocking probability, which can be expressed as the probability of having more than $W_{l}\left(W_{l}\right.$ : capacity of link $\left.l\right)$ connections in ON state. Given that the probability of a connection being in ON state corresponds to a Bernoulli random variable with parameter $\rho$, the probability of having $n$ connections in $\mathrm{ON}$ state corresponds to a Binomial random variable with parameters $N_{l}$ (number of connections whose routes use link $l$ ) and $\rho$ (probability of a connection being in ON state). Thus, the link blocking probability can be estimated from:

$$
B_{l}=1-\sum_{n=0}^{W_{l}}\left(\begin{array}{c}
N_{l} \\
n
\end{array}\right) \rho^{n}(1-\rho)^{N_{l}-n}
$$

By combining Eqs. (4) and (5), $W_{l}$ is determined as the minimum number that satisfies:

$$
1-\sum_{n=0}^{W_{l}}\left(\begin{array}{c}
N_{l} \\
n
\end{array}\right) \rho^{n}(1-\rho)^{N_{l}-n} \leq 1-\sqrt[\hat{H}_{l}]{(1-B)}
$$

where the left side corresponds to the estimation of the actual link blocking as a function of $W_{l}$ whilst the right side corresponds to the target value of the link blocking.

\subsubsection{Integer linear optimisation model}

The proposed optimisation method (for minimum wavelength requirements) operates as follows:

1. Determine the set $S$ of all possible different routes, considering all node pairs. For the ring topology there are only two different routes per node pair; thus, the cardinality of $S$ is equal to $2 N(N-1)$.

2. Given the set $S$, an integer programming model is used to determine an optimal route (in the sense that the sum of the number of wavelengths required at each link 
in the network is minimised) for each node pair. The integer programming model utilises the following notation:

Indexes and Parameters. Let

- $\quad i$ and $j$ be the indexes for nodes in $\mathcal{N}$,

- $\quad l$ be the index for a link in $\mathcal{L}$,

- $\quad R_{i, j, k}$ be the k-th route connecting the source node $i$ to the destination node $j$. If $k=0$, the counter-clockwise route is used; if $k=1$ the clockwise route is selected.

- $\quad \delta_{i, j, k, l}$ be the input parameter that takes the value 1 if the $k$-th route $(0 \leq k$ $\leq 1)$ connecting the source node $i$ to the destination node $j(i \neq j)$ uses link $l$; and 0 otherwise.

Decision variables. Let

- $\quad \alpha_{i, j, k}$ be a binary decision variable that takes the value 1 if the $k$-th route $(0 \leq k \leq 1)$ connecting the node $i$ to node $j(i \neq j)$ is used by the routing algorithm; and 0 otherwise

- $\quad N_{l}$ be an integer decision variable that denotes the number of routes that use link $l$, given by Eq. (10) below.

The integer programming model can be described as follows:

$$
\operatorname{Min} C=\sum_{l \in \mathcal{L}} W_{l}
$$

subject to:

$$
\begin{array}{ll}
\alpha_{i, j, k} \in\{0,1\} & ; \text { for all } i \in \mathcal{N}, j \in \mathcal{N}, k \in\{0,1\} \\
\sum_{k=0}^{1} \alpha_{i, j, k}=1 & ; \text { for all } i \in \mathcal{N} \text { and } j \in \mathcal{N} \quad(i \neq j) \\
N_{l}=\sum_{\forall i \neq j k=0} \sum_{i, j, k, l}^{1} \alpha_{i, j, k} & ; \text { for all } l \in \mathcal{L}
\end{array}
$$

Eq. (7) is evaluated using the values of $W_{l}$ obtained from the routing algorithm and Eq. (6). Constraint (8) imposes the 0-1 value of variables $\alpha_{i, j, k}$ (as stated in the definition of this variable); constraint (9) imposes that only one route connecting nodes $i$ and $j$ must be used and constraint (10) determines the value of the integer variable $N_{l}$.

\section{Numerical Results}

The integer programming model (7)-(10) was solved on a PC Pentium IV (3192 MHz. and 1GB RAM). The algebraic modelling language AMPL was used, with CPLEX 10.0 as solver [18]. 
The optimal routing and dimensioning method described in section 2 was applied to rings of different sizes under different values of traffic load $(\rho)$ with a target $B_{l}$ of $10^{-6}$. The wavelength requirements were compared to those obtained with Eq. (6) when applying the balanced-load routing algorithm proposed in [8]. The algorithm in [8] determines the routes which minimise the wavelength requirements of WDM networks whilst balancing the traffic load (which has been the common approach for network dimensioning).

Table 1 shows the total wavelength requirements obtained with the method proposed in this paper and the balancing load method of [8] for rings from 6 to 16 nodes (in the table, P.M. and B.L. stand for "proposed method" and "balanced load", respectively) for different values of traffic load. Fig. 1 shows graphically these results for rings of $6,8,12$ and 16 nodes. The cases where the proposed method requires a number of wavelengths lower than the balanced-load approach have been highlighted in bold in Table 1. In the remaining entries in Table 1 the proposed optimisation method achieves the same cost as the balanced-load approach.

Table 1. Wavelength requirements for rings of 6-16 nodes for the proposed method and the balanced-load approach

\begin{tabular}{|c|c|c|c|c|c|c|c|c|c|c|c|}
\hline$\rho$ & $\begin{array}{c}\mathbf{N}=\mathbf{6} \\
\mathbf{P M} / \mathbf{B L}\end{array}$ & $\begin{array}{c}\mathbf{N}=7 \\
\mathbf{P M} / \mathbf{B L}\end{array}$ & $\begin{array}{c}\mathbf{N}=\mathbf{8} \\
\mathbf{P M} / \mathbf{B L} \\
\end{array}$ & $\begin{array}{c}\mathbf{N}=\mathbf{9} \\
\mathbf{P M} / \mathbf{B L}\end{array}$ & $\begin{array}{c}\mathbf{N}=10 \\
\mathbf{P M} / \mathbf{B L}\end{array}$ & $\begin{array}{c}\mathrm{N}=11 \\
\mathrm{PM} / \mathbf{B L}\end{array}$ & $\begin{array}{c}\mathrm{N}=12 \\
\mathbf{P M} / \mathbf{B L}\end{array}$ & $\begin{array}{c}\mathbf{N}=13 \\
\mathbf{P M} / \mathbf{B L} \\
\end{array}$ & $\begin{array}{c}\mathrm{N}=14 \\
\mathrm{PM} / \mathbf{B L} \\
\end{array}$ & $\begin{array}{c}\mathbf{N}=15 \\
\mathbf{P M} / \mathbf{B L} \\
\end{array}$ & $\begin{array}{c}\mathrm{N}=16 \\
\mathrm{PM} / \mathbf{B L}\end{array}$ \\
\hline 0.1 & $48 / 54$ & $69 / 70$ & $92 / 96$ & $117 / 126$ & $156 / 160$ & $187 / 198$ & $228 / 234$ & $273 / 286$ & $321 / 322$ & $360 / 360$ & $416 / 416$ \\
\hline 0.2 & $54 / 54$ & $82 / 84$ & $116 / 128$ & $153 / 162$ & $200 / 210$ & $250 / 264$ & $300 / 306$ & $364 / 364$ & $434 / 434$ & $510 / 510$ & $592 / 608$ \\
\hline 0.3 & $54 / 54$ & $84 / 84$ & $127 / 128$ & $171 / 180$ & $227 / 230$ & $286 / 286$ & $358 / 366$ & $430 / 442$ & $518 / 518$ & $627 / 630$ & $722 / 736$ \\
\hline 0.4 & $54 / 54$ & $84 / 84$ & $128 / 128$ & $180 / 180$ & $244 / 250$ & 314/330 & $396 / 402$ & $482 / 494$ & $588 / 588$ & $690 / 690$ & $816 / 832$ \\
\hline 0.5 & $54 / 54$ & $84 / 84$ & $128 / 128$ & $180 / 180$ & $250 / 250$ & $329 / 330$ & $420 / 432$ & $520 / 520$ & $634 / 644$ & $768 / 780$ & \begin{tabular}{|l|}
$912 / 928$ \\
\end{tabular} \\
\hline 0.6 & $54 / 54$ & $84 / 84$ & $128 / 128$ & $180 / 180$ & $250 /$ & $330 / 330$ & $432 / 432$ & $546 / 546$ & $672 /$ & 810 & 992 \\
\hline 0.7 & $54 / 54$ & $84 / 84$ & $128 / 128$ & $180 /$ & 250 & 330 & $432 / 432$ & $546 / 546$ & 686 & 840 & $1020 / 1024$ \\
\hline 0.8 & $54 / 54$ & $84 / 84$ & $128 / 128$ & $180 / 180$ & $250 / 250$ & $330 / 330$ & $432 / 432$ & $546 / 546$ & $686 /$ & $840 / 840$ & $1024 / 1024$ \\
\hline 0.9 & $54 / 54$ & $84 / 84$ & $128 / 128$ & $180 / 180$ & $250 / 250$ & $330 / 330$ & $432 / 432$ & $546 / 546$ & $686 / 686$ & $840 / 840$ & $\mid 1024 / 1024$ \\
\hline
\end{tabular}

It can be seen that, depending on the value of the traffic load, the optimisation method proposed in this paper requires a lower or equal number of wavelengths than the usual approach of determining the routes by means of load balancing. Results show that the range of traffic load values (in steps of 0.1 ) at which the routing and dimensioning method proposed here achieves wavelength savings with respect to the balanced approach increases with the ring size (in number of nodes): for the 6-node ring a saving of 6 wavelengths was achieved for only one value of the traffic load $(0.1)$ whilst for the16-node ring savings of up to 16 wavelengths were observed in the range [0.1- 0.7]. As most networks currently operate at low loads (typically $<0.3$ [ 19 , 20]), the proposed method will ensure benefits where it is needed most.

It can also be seen that the load-balanced approach is optimal for small-size rings ( $<8$ nodes) in a wide range of traffic loads as well as for high loads $(>0.7)$ in all the studied rings.

As a way of example, Fig. 2 shows the routing matrices of the proposed method (upper left) and the balanced-load approach (upper right) for the 8-node ring with a traffic load of 0.1 . In each matrix the element $(i, j)$ corresponds to the parameter $k$ defined in section 2.2. The elements in the matrix of the proposed method which are different from the balanced-load approach are highlighted in bold. 

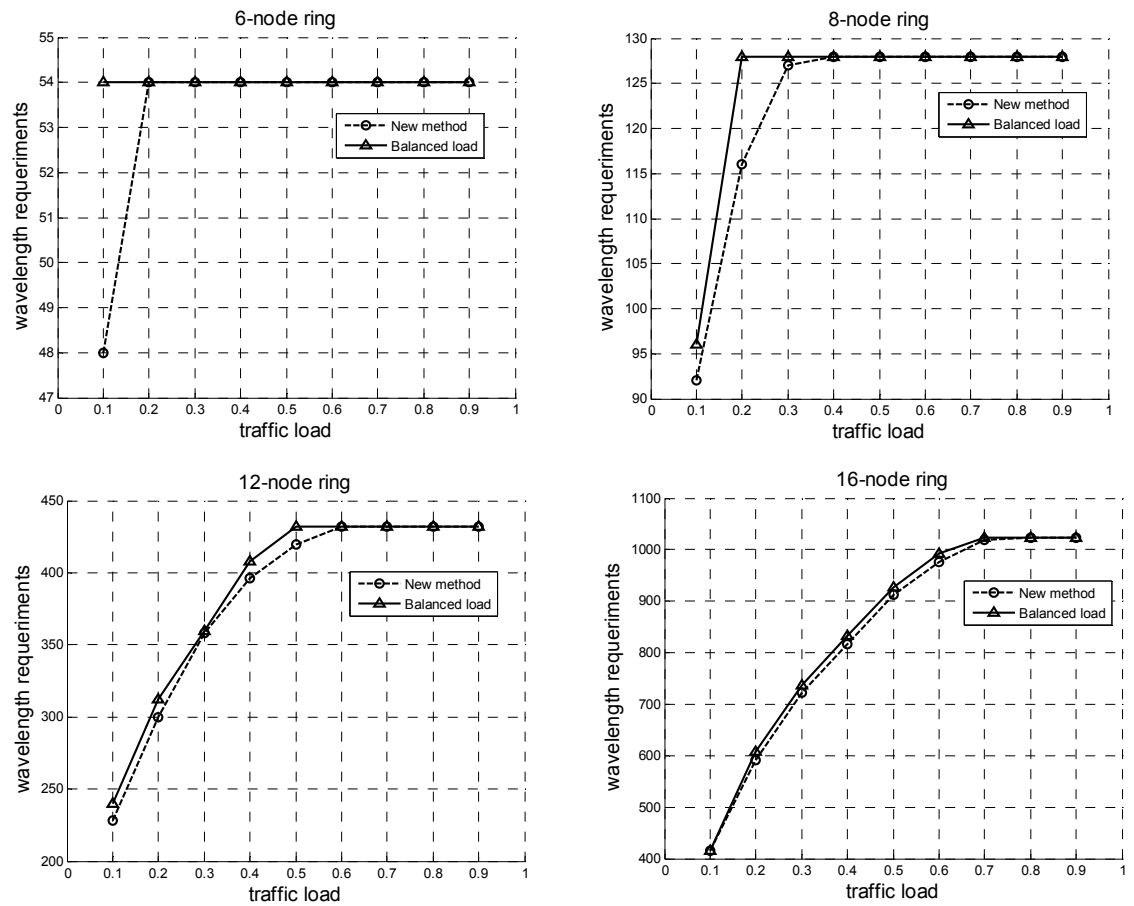

Fig. 1. Wavelength requirements for rings of 6 (upper left), 8 (upper right), 12 (lower left) and 16 (lower right) nodes using balanced-load routing and the method proposed in this paper for $\rho=0.1$

\begin{tabular}{|c|c|c|c|c|c|c|c|}
\hline 1 & 2 & 3 & 4 & 5 & 6 & 7 & 8 \\
\hline 1 & 1 & 1 & 1 & 1 & 1 & 1 & 0 \\
\hline 0 & - & 1 & 1 & 1 & 1 & 1 & 0 \\
\hline 1 & 0 & - & 1 & 1 & 1 & 1 & 1 \\
\hline 1 & 0 & 0 & - & 1 & 1 & 1 & 1 \\
\hline 1 & 1 & 0 & 0 & - & 1 & 1 & 1 \\
\hline 1 & 1 & 1 & 1 & 0 & - & 1 & 1 \\
\hline 1 & 1 & 1 & 1 & 0 & 0 & - & 1 \\
\hline 1 & 1 & 1 & 1 & 1 & 0 & 0 & - \\
\hline
\end{tabular}

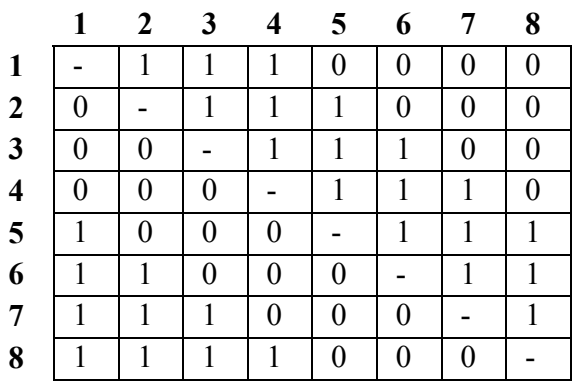

Fig.2. Routing matrix (1 denotes the route in clockwise direction; 0 in counter-clockwise direction) for the proposed method (left) and the balanced-load approach (right) for a 8-node ring with a traffic load equal to 0.1

Table 2 shows, for the same case (8-node ring, $\rho=0.1$ ), the number of connections per link $\left(N_{l}\right)$ obtained by applying the optimisation method (whereas in the load-balanced approach, $N_{l}=8$ for each link). 
Table 2. Value of $N_{l}$ in a 8 -node ring. Routing determined by the optimisation method for $\rho=0.1$

\begin{tabular}{|l|c|c|c|c|c|c|c|c|}
\cline { 2 - 9 } \multicolumn{1}{c|}{} & \multicolumn{7}{c|}{ Clockwise links (“i->j” denotes the link from node $i$ to node $\boldsymbol{j}$ ) } \\
\cline { 2 - 9 } \multicolumn{1}{c|}{} & $1->2$ & $2->3$ & $3->4$ & $4->5$ & $5->6$ & $6->7$ & $7->8$ & $8->1$ \\
\hline$N_{l}$ & 17 & 17 & 18 & 17 & 17 & 18 & 17 & 17 \\
\hline \multirow{4}{*}{} & $2->1$ & $3->2$ & $4->3$ & $5->4$ & $6->5$ & $7->6$ & $8->7$ & $1->8$ \\
\hline & 2 & 2 & 3 & 2 & 2 & 3 & 2 & 2 \\
\hline
\end{tabular}

From Fig. 2 and Table 2 it can be observed that the proposed method concentrated as many connections as possible in the same link (by using longer routes if necessary) as long as the statistical gain of doing so was higher than the extra wavelengths required by the longer routes. See, for example, the route connecting the node 1 to the node 6 (from Fig.2): whilst under a balanced-load approach the shortest route is used (i.e. the route made of the nodes 1-8-7-6) in the optimal method proposed here a longer route (made of the nodes 1-2-3-4-5-6) is preferable as the statistical gain is higher.

\section{Conclusions}

In this paper a new routing and link dimensioning optimisation method was proposed. The method aims to minimise the wavelength requirements of dynamic end-to-end OBS ring networks by solving a linear integer optimisation model for the routing problem. Unlike previous approaches, the method does not necessarily balance the traffic load. In fact, obtained results show that in some cases the method does exactly the opposite: it concentrates the load (by using routes longer than the shortest paths if necessary) as long as the additional number of wavelengths due to the longer paths is lower than the number of wavelengths saved due to the higher statistical gain.

The method can be implemented in dynamic optical rings by storing the optimal set of routes (obtained in an off-line manner) in a routing table from which the route information for a specific node pair can be retrieved on demand.

The proposed method was applied to ring networks from 6 to 16 nodes. It was found that, compared to the balanced approach, the proposed method could achieve wavelength savings of up to 16 wavelengths and that the wavelength savings increased with the ring size (in number of nodes). It was also observed that the balanced-load approach is optimal for small-size rings ( $<8$ nodes) in a wide range of traffic loads as well as for high loads $(>0.7)$ in all the studied rings.

These results show that the usual approach of balancing the load in rings does not always yield the optimal results in terms of wavelength requirements and that by applying a selective unbalancing of load wavelength savings can be achieved.

Acknowledgements. Financial support from Fondecyt Project \#1050361 (Chilean Government) and USM Projects 23.07.27, 23.07.28 and 28.06.21 is gratefully acknowledged. We would also like to thank Marco Tarifeño for collecting and formatting the results of the optimisation program. 


\section{References}

1. Qiao, C., Yoo, M.: Optical Burst Switching (OBS) - a new paradigm for an optical internet. Journal of High Speed Networks, Vol.8 (1999) 69-84

2. Turner, J.: Terabit burst switching. Journal of High Speed Networks, Vol.8 (1999) 3-16

3. Düser, M., Bayvel, P.: Analysis of a dynamically wavelength-routed optical burst switched network architecture. J. of Lightwave Technology, Vol.4 (2002) 574-585

4. Arakawa, S., Miyamoto, K., Murata, M., Miyahara, H.: Delay analyses of wavelength reservation methods for high-speed burst transfer in photonic networks. In Proc. International Technical Conference on Circuits and Systems, Computers and Comms. (ITCCSCC99) July 1999

5. Zapata, A., de Miguel, I., Dueser, M., Spencer, J., Bayvel, P., Breuer, D., Hanik, N., Gladish, A.: Next Generation 100-Gigabit Metro Ethernet (100GbME) using multiwavelength optical rings. IEEE/OSA Journal of Lightwave Technology, special issue on Metro \& Access Networks, Vol. 22 (11), (2004), 2420-2434

6. Zapata A., Bayvel P.: Optimality of resource allocation algorithms in dynamic WDM networks. In Proceedings of $10^{\text {th }}$ European Conference on Network and Optical Communications, NOC 2005, London, UK, 5-7 July 2005, pp.131-138

7. Baroni, S.; Bayvel, P.: Wavelength requirements in arbitrarily connected wavelength-routed optical networks. IEEE/OSA Journal of Lightwave Technology, Vol. 5 (2), (1997), 242-251

8. Hunter, D.; Marcenac, D.: Optimal mesh routing in four-fibre WDM rings. Electronic Letters, Vol. 34 (8), (1998), 796-797

9. Van Parys, W.; Van Caenegem, B.; Demeester, P.: Reduction of blocking in arbitrary meshed WDM networks through a biased routing approach. Proceedings Optical Fiber Communicactions Conference, OFC'98, San José, CA, USA, page 94

10. Zapata, A.; Bayvel, P.: Improving the scalability of lightpath assignment algorithms in dynamic networks. Proceedings $31^{\text {st }}$ European Conference on Optical Communications, ECOC 2005, Glasgow, Scotland, September 2005

11. Späth, J.: Dynamic routing and resource allocation in WDM transport networks. Computer Networks 32 (2000), 519-538

12. Ramaswami, R.: Optical networking technologies: what worked and what didn't. IEEE Communications Magazine, v.44 (9), 132-139, September 2006

13. Choi, J. Y., Vu, H.L., Cameron, C. W., Zukerman, M., Kang, M.: The effect of burst assembly on performance of optical burst switched networks. Lecture Notes on Computer Science, Vol.3090 (2004) 729-739

14. Tancevski, L., Bononi, A., Rusch, L. A.: Output power and SNR swings in cascades of EDFAs for circuit- and packet-switched optical networks. Journal of Lightwave Technology, Vol.17 (5) (1999) 733-742

15. Zukerman, M., Wong, E., Rosberg, Z., Lee, G., Lu, H.V.: On teletraffic applications to OBS. IEEE Communications Letters, Vol.8 (2) (2004) 731-733

16. Adas A: Traffic models in broadband networks, IEEE Communications Magazine, vol. 35, no. 7, (July 1997), pp. 82-89

17. Ross S.: Introduction to probability models. $6^{\text {th }}$ Edition Academic Press, 1997, (Eq. 7.15)

18. Fourer, R.; Gay, D.M.; Kernigham, B.W.: AMPL: a modeling language for mathematical programming. $2^{\text {nd }}$ ed. The Scientific Press, USA, 2000.

19. Odlyzko, A.: Data networks are lightly utilized, and will stay that way. Review of Network Economics, Vol. 2, (2003), 210-237

20. Bhattacharyya, S.; Diot, C.; Jetcheva, J.; Taft, N.: POP-Level and access-link-level traffic dynamics in a Tier-1 POP. ACM SIGCOMM Internet Measurement Workshop, Vol. 1, 3953, San Francisco, CA, November 2001 